\title{
Valor preditivo da medida da cintura e da relação cintura-quadril no diagnóstico do diabetes melito e da dislipidemia*
}

\author{
Predictive value of the waist measurement and the \\ waist-hip-ratio relationship on the diagnosis of \\ diabetes mellitus and dyslipidemia*
}

\begin{abstract}
Ana Luísa Garcia Calich ${ }^{\star *}$, André Russowsky Brunoni ${ }^{\star *}$, Renato Mansini ${ }^{\star *}$, Fábio Rodrigues Ferreira do Espírito Santo**, Isabela M. Benseñor ${ }^{\star \star \star}$
\end{abstract}

Calich ALG, Brunoni AR, Mansini R, Santo FRPE, Benseñor IM. Valor preditivo da medida da cintura e da relação cintura-quadril no diagnóstico do diabetes melito e da dislipidemia. Rev Med (São Paulo) 2002 jan./dez.;81(1/4):8-14.

RESUMO: Introdução: O índice de obesidade está aumentando no Brasil.A medida da cintura (MC) é uma boa medida para a avaliação da obesidade localizada e é mais fácil de ser adquirida do que a relação cintura quadril (RCQ). Entretanto, os efeitos da obesidade regional foram melhores descritos entre a RCQ e fatores de risco cardiovasculares. Objetivo: testar se a medida da cintura isolada é melhor do que a RCQ para predizer diabetes e dislipidemia. Métodos: analisamos a razão de verossimilhança positiva e negativa da MC e RCQ numa amostra de 202 homens e mulheres com idade variando de 20 a 74 anos e aparentemente saudáveis como fator preditivo para diabetes e dislipidemia. Resultados: (1) Para mulheres, a RCQ foi melhor fator preditivo para o diagnostico dediabetes $\left(R V_{+}=2,5\right.$ e $\left.R V-=0,2\right)$ e dislipidemia $(R V+2,5$ e $R V-=0,2)$ comparado a $M C$ (diabetes, $R V+=1,9$ e $R V-=0,4$; dislipidemia, $\mathrm{RV}+=1,6$ e RV- = 0,6); (2) Para homens, a $\mathrm{MC}$ foi melhor fator preditivo para o diagnostico de diabetes $\left(R V_{+}=2,4\right.$ e $\left.R V-=0,6\right)$ e dislipidemia $\left(R V_{+}=5\right.$ e $\left.R V-=0,5\right)$ do que a $R C Q$ (diabetes, $R V+=1,0$ e $R V$ - = 0,9; dislipidemia, $R V_{+}=2,1$ e $\left.R V-=0,7\right)$. Conclusão: $A M C$ e a $R C Q$ se comportam diferentes entre homens e mulheres.

DESCRITORES: Diabetes mellitus/diagnóstico; Hiperlipidemia/diagnóstico; Valor preditivo; Constituição corporal; Obesidade/etiologia.

\section{INTRODUÇÃO}

A obesidade é uma alteração bastante prevalente em muitos países desenvolvidos e está freqüentemente associada com outras doenças como diabetes melito, hipertensão arterial, dislipidemias e doenças cardiovasculares. Entretanto, a prevalência da obesidade também é um importante problema de saúde publica emergente em países como o Brasil que passam pela chamada transição epidemiológica com crescente predomínio das doenças crônicas como principal causa de morbimortalidade ${ }^{8}$.

* Prêmio Oswaldo Cruz (Área Medicina Preventiva) - XXI COMU Congresso Médico Universitário da FMUSP.

** Acadêmicos da FMUSP.

*** Preceptora de Clínica Médica do HC-FMUSP.

Endereço para correspondência: Ana Luísa Calech, Rua Tabapuã, 1590 Apto. 42 - CEP: 04533-005 São Paulo, SP. 
Dados recentes, obtidos a partir de três inquéritos probabilísticos realizados nas últimas três décadas no Brasil, mostram as diferenças distribuição da obesidade entre as regiões Sudeste (a mais rica) e Nordeste (a mais pobre) do Brasil considerando-se como ponto de corte um índice de massa corpórea (IMC) acima de $30 \mathrm{~kg} / \mathrm{m}^{2}$. No período de 1975-1989, há um aumento da prevalência de obesidade tanto na região Nordeste quanto na Sudeste, com uma maior proteção da região Nordeste em função das condições socioeconômicas da população incluindo homens e mulheres. No período de 1889-1997, essas características persistem para a população masculina, com um maior aumento da freqüência de obesidade na região Nordeste em relação à Sudeste, com diminuição das diferenças entre as duas regiões. Entretanto, para a população feminina ocorre um aumento da obesidade nitidamente influenciado pela renda familiar de forma inversa nas duas regiões: enquanto na região Nordeste o aumento da prevalência de obesidade concentrou-se no estratos intermediários e de alta renda, na região Sudeste, houve um declínio da prevalência de obesidade nos estratos intermediários e de alta renda. Esse padrão de comportamento da obesidade nas mulheres de mais alta renda da região Sudeste somente é comparável ao comportamento de algumas populações escandinavas, não tendo sido detectado em outros países em desenvolvimento ${ }^{11}$.

Estudo recente, em São Paulo, mostrou uma maior concentração de sobrepeso e obesidade central em mulheres de baixa estatura do que em mulheres com estatura normal. Essas diferenças não foram observadas para o sexo masculino ${ }^{17}$.

Nos estudos de obesidade, o Índice de Massa Corpórea (IMC) é frequentemente considerado o padrão ouro para o diagnóstico da doença. Entretanto, avaliar a distribuição da gordura corpórea é importante para estimar os fatores de risco de doenças cardiovasculares. A obesidade abdominal indicada por uma relação cintura-quadril (RCQ) elevada tem sido mostrada como um fator preditivo, independente do IMC para muitas doenças como hipertensão arterial, doença coronária, diabetes melito não insulino dependente e infarto agudo do miocárdio ${ }^{18}$. Isso provavelmente ocorre devido a mudanças metabólicas associadas com o deposito de gordura intraabdominal ${ }^{9}$.

Numerosos estudos demonstraram que homens e mulheres com valores elevados da RCQ apresentavam maior risco de morte, síncope e miocardiopatia isquêmica, de intolerância a glicose, bem como de níveis mais elevados de pressão arterial e de lipides séricos ${ }^{3,5,6}$. Entretanto, a medida da RCQ não é precisa pois o ponto exato para a obtenção da medida do quadril não é anatomicamente bem definido.

Recentemente, devìlo a dificuldade na obtenção exata da medida da circunferência do quadril, tentou-se substituir a relação cintura-quadril pela simples aferição da circunferência da cintura (CC) de mais fácil obtenção e também correlacionada a outros fatores de risco para doença cardiovascular, $7,9,10,14$.

O objetivo desse trabalho é avaliar se a medida da circunferência da cintura é tão precisa quanto a medida da relação cintura quadril como fator preditivo para o aparecimento de diabetes melito e um elevado índice da relação entre colesterol total e colesterol HDL e qual desses índices melhor se correlacionaria com essas doenças na população brasileira.

\section{MÉTODOS}

\section{Pacientes}

Foram estudados 212 pacientes que procuraram espontaneamente o Hospital das Clínicas da Faculdade de Medicina da Universidade de São Paulo, entre fevereiro e dezembro de 2000, para uma consulta de promoção a saúde no ambulatório de Clínica Médica do Hospital das Clínicas, as segundas-feiras à tarde, horário que os alunos tinham disponível para o atendimento.

Para cada paciente foi feita uma anamnese e exame físico padrão. Doenças diagnosticadas foram tratadas e houve seguimento dos pacientes até que as doenças diagnosticadas fossem resolvidas.

\section{Medidas antropométricas}

Coletou-se dados referentes à altura, peso, medida da cintura e medida do quadril em todos os pacientes avaliados. A medida da cintura foi definida como a circunferência de menor diâmetro entre o gradeado costal e a cicatriz umbilical e a medida do quadril como a maior circunferência no nível do grande trocanter do fêmur ${ }^{13}$.

O IMC foi calculado dividindo-se o peso pela altura ao quadrado $\left(\mathrm{kg} / \mathrm{m}^{2}\right)$ sendo classificado de acordo com os critérios da OMS. A RCQ foi calculada dividindo a medida da cintura pela medida do quadril e definiuse um valor elevado quando a RCQ era e $\geq 0,95$ nos homens e $R C Q$ e $\geq 0,85$ nas mulheres.

A MC foi definida como elevada quando ultrapassava $100 \mathrm{~cm}$ para homens e $85 \mathrm{~cm}$ para mulheres ${ }^{19}$.

\section{DIAGNÓSTICOS}

O diagnóstico de diabetes melito foi definido como nível de glicemia acima de $126 \mathrm{mg} / \mathrm{dl}$ e o de dislipidemia como uma relação colesterol total sobre HDL-colesterol acima de $5^{2,15}$. 


\section{Dosagem da glicemia}

Para dosagem da glicemia foi utilizado o método da hexoquinase ${ }^{1}$.

\section{Dosagem do colesterol total}

Para a dosagem do colesterol total será utilizado o kit COBAS INTEGRA Cholesterol. Trata-se de método enzimático colorimétrico ( $\mathrm{CHOD} / \mathrm{PAP}$ ) com colesterol esterase, colesterol oxidase e 4-aminoantipirina. Cada kit faz 400 exames. Valores normais para o sexo feminino são de 5,4 a $24,5 \mathrm{U} / \mathrm{L}$ $(0,09$ a $0,41 \mathrm{mKat} / \mathrm{L})$ e para o sexo masculino de $8,2 \mathrm{a}$ $38,1 \mathrm{U} / \mathrm{L}(0,14 \text { a } 0,68 \mathrm{mKat} / \mathrm{L})^{12}$.

\section{Dosagem do HDL-colesterol}

Para a dosagem do HDL-colesterol será utilizado o kit COBAS INTEGRA Cholesterol com reagente separado para HDL-colesterol. A concentração do HDL é determinada após isolamento do HDL-colesterol na amostra. O reagente de separação para o HDL utiliza ácido fosfotúngstico e íons de magnésio para precipitar os quilomicrons, o VLDL e o LDL. Após centrifugação, o HDL que permanece no sobrenadante é quantificado através do seu conteúdo em colesterol. A concentração de HDLcolesterol é determinada por intermédio de um método enzimático colorimétrico. Cada kit faz 400 exames. Valores normais para o sexo feminino 45 a $65 \mathrm{mg} / \mathrm{dL}$ $(1,2$ a $1,7 \mathrm{mmol} / \mathrm{L})$ e para o sexo masculino 35 a $55 \mathrm{mg} / \mathrm{dL}(0,9 \text { a } 1,4 \mathrm{mmol} / \mathrm{L})^{16}$.

\section{Dosagem de triglicérides}

Para a dosagem de triglicérides será utilizado o kit COBAS INTEGRA Triglycerides. Trata-se de método enzimático colorimétrico (GPO/PAP) com glicerol fosfato oxidase e 4-aminoantipirina. Cada kit faz 300 exames. Valores normais para o sexo feminino de 35 a $135 \mathrm{mg} / \mathrm{dL}(0,40$ a $1,54 \mathrm{mmol} / \mathrm{L})$ e para o sexo masculino de 40 a $160 \mathrm{mg} / \mathrm{dL}(0,45 \text { a } 1,82 \mathrm{mmol} / \mathrm{L})^{16}$.

\section{ANÁLISE ESTATÍSTICA}

Calculou-se a sensibilidade, especificidade, os valores preditivos positivo e negativo da medida da cintura e da relação cintura-quadril para o diagnóstico de diabetes melito e dislipidemia. Também foram calculados os valores da razão de verossimilhança positiva e negativa da medida da cintura e da relação cintura quadril para os mesmos diagnósticos ${ }^{4}$.

\section{RESULTADOS}

Foram analisados 212 pacientes dos quais $75 \%$ eram do sexo feminino. Essa é a porcentagem normal de pacientes do sexo feminino no Ambulatório Geral e Didático. A Tabela 1 mostra as diferenças entre a média dos valores de peso, altura, IMC, medida da cintura, relação cintura-quadril, glicemia, colesterol total e frações para homens e mulheres na amostra.

Tabela 1 - Características gerais da população estudada distribuídas por sexo.

\begin{tabular}{l|l|l|l}
\hline & & Homens & Mulheres \\
\hline \multirow{2}{*}{ Idade (anos) } & Média & 36,2 & 37,7 \\
& Desvio padrão & 15,7 & 13,6 \\
\hline \multirow{2}{*}{ Altura (m) } & Média & 1,7 & 2,6 \\
& Desvio padrão & 7,8 & 13,3 \\
\hline \multirow{2}{*}{ iMC kg/m2) } & Média & 25,7 & 26,9 \\
& Desvio padrão & $7,39,7$ & \\
\hline \multirow{2}{*}{ (molesterol total } & Média & 183,1 & 184,2 \\
& Desvio padrão & 43,3 & 39,4 \\
\hline \multirow{2}{*}{ HDL (mg/dl) } & Média & 46,2 & 54,3 \\
& Desvio padrão & 13,0 & 13,0 \\
\hline \multirow{2}{*}{ LDL (mg/dl) } & Média & 111,5 & 111,4 \\
& Desvio padrão & 39,3 & 36,8 \\
\hline
\end{tabular}

As Tabelas 2 e 3 mostram os valores da sensibilidade, especificidade, valor preditivo positivo, valor preditivo negativo e razão de verossimilhança positiva e negativa da medida da cintura e da relação cintura-quadril na população feminina para o diagnóstico de diabetes melito. Os valores de sensibilidade, especificidade, valor preditivo positivo e valor preditivo negativo foram mais elevados para a relação cintura-quadril em relação à medida isolada da cintura para o diagnóstico de diabetes melito. Isso se confirma por uma razão de verossimilhança positiva mais elevada e uma razão de verossimilhança mais próxima do zero para a relação cintura-quadril.

Tabela 2 - Valor da sensibilidade, especificidade, valor preditivo positivo e valor preditivo negativo, razão de verossimilhança positiva e razão de verossimilhança negativa da medida da cintura para diagnóstico de diabetes nas mulheres.

\begin{tabular}{l|c|c|c}
\hline & & \multicolumn{2}{|c}{ Diabetes melito } \\
\hline & & Presente & Ausente \\
\hline $\begin{array}{l}\text { Medida da } \\
\text { cintura (cm) }\end{array}$ & Elevada & 6 & 50 \\
\cline { 2 - 4 } & Normal & 2 & 74 \\
\hline
\end{tabular}

Sensibilidade $=75 \%$; Especificidade $=60 \%$; Valor Preditivo positivo $=12 \%$; Valor preditivo negativo $=97,3 \%$; Razão de verossimilhança positiva $=1,9$; Razão de verossimilhança negativa $=0,42$. 
Tabela 3 - Valor da sensibilidade, especificidade, valor preditivo positivo e valor preditivo negativo, razão de verossimilhança positiva e razão de verossimilhança negativa da medida da relação cintura-quadril para diagnóstico de diabetes nas mulheres.

\begin{tabular}{l|c|c|c}
\hline & & \multicolumn{2}{|c}{ Diabetes melito } \\
\hline & & Presente & Ausente \\
\hline \multirow{2}{*}{$\mathrm{RCQ}$} & Elevada & 7 & 44 \\
\cline { 2 - 4 } & Normal & 1 & 80 \\
\hline
\end{tabular}

Sensibilidade $=88 \%$; Especificidade $=65 \%$; Valor Preditivo positivo = 14\%; Valor preditivo negativo = 99,3\%; Razão de verossimilhança positiva $=2,51$; Razão de verossimilhança negativa $=0,19$.

As Tabelas 4 e 5 mostram os valores da sensibilidade, especificidade, valor preditivo positivo e valor preditivo negativo da medida da cintura e da relação cintura-quadril para o diagnóstico de dislipidemia. Novamente os resultados obtidos em relação a RCQ são superiores aos resultados obtidos com a medida isolada da cintura nas mulheres. Os valores da razão de verossimilhança positiva são também mais elevados e os valores da razão de verossimilhança negativa mais próximos do zéro para a relação cintura-quadril comparada a medida da cintura isolada.

Tabela 4 - Valor da sensibilidade, especificidade, valor preditivo positivo e valor preditivo negativo, razão de verossimilhança positiva e razão de verossimilhança negativa da medida da cintura para diagnóstico de dislipidemia nas mulheres.

\begin{tabular}{l|c|c|c}
\hline & & \multicolumn{2}{|c}{$\begin{array}{c}\text { Dislipidemia } \\
\text { (Razão CT/HDL) }\end{array}$} \\
\hline & & Aumentada (>5) & Normal (<5) \\
\hline $\begin{array}{l}\text { Medida da } \\
\text { cintura (cm) }\end{array}$ & Elevada & 9 & 46 \\
\cline { 2 - 4 } & Normal & 5 & 70 \\
\hline
\end{tabular}

Sensibilidade $=64 \%$; Especificidade $=60 \%$; Valor Preditivo positivo $=17 \%$; Valor preditivo negativo $=93,3 \%$; Razão de verossimilhança positiva $=1,6$; Razão de verossimilhança negativa $=0,60$.

Tabela 5 - Valor da sensibilidade, especificidade, valor preditivo positivo e valor preditivo negativo, razão de verossimilhança positiva e razão de verossimilhança negativa da medida da relação cintura-quadril para diagnóstico de dislipidemia nas mulheres.

\begin{tabular}{c|c|c|c}
\hline & & \multicolumn{2}{|c}{$\begin{array}{c}\text { Dislipidemia } \\
\text { (Razão CT/HDL) }\end{array}$} \\
\hline & & Aumentada (> 5) & Normal (<5) \\
\hline \multirow{2}{*}{ RCQ } & Elevada & 12 & 39 \\
\cline { 2 - 4 } & Normal & 2 & 77 \\
\hline
\end{tabular}

Sensibilidade $=86 \%$; Especificidade $=66 \%$; Valor Preditivo positivo $=24 \%$; Valor preditivo negativo $=97,4 \%$; Razão de verossimilhança positiva $=2,5$; Razão de verossimilhança negativa $=0,21$.
As Tabelas 6 e 7 mostram os valores da sensibilidade, especificidade, valor preditivo positivo e valor preditivo negativo da medida da cintura e da relação cintura-quadril para o sexo masculino. Os valores obtidos para a medida da cintura foram superiores aos valores obtidos para relação cinturaquadril na amostra masculina. Os valores da razão de verossimilhança positiva são mais elevados e a razão de verossimilhança negativa mais próxima do zéro para a medida da cintura em relação à $R C Q$ na amostra masculina.

Tabela 6 - Valor da sensibilidade, especificidade, valor preditivo positivo e valor preditivo negativo, razão de verossimilhança positiva e razão de verossimilhança negativa da medida da cintura para diagnóstico de diabetes nos homens.

\begin{tabular}{l|c|c|c}
\hline & & \multicolumn{2}{|c}{ Diabetes melito } \\
\hline & & Presente & Ausente \\
\hline Medida da & Elevada & 2 & 8 \\
\cline { 2 - 4 } cintura $(\mathrm{cm})$ & Normal & 2 & 30 \\
\hline
\end{tabular}

Sensibilidade $=50 \%$; Especificidade $=79 \%$; Valor Preditivo positivo $=20 \%$; Valor preditivo negativo $=94 \%$; Razão de verossimilhança positiva $=2,4$; Razão de verossimilhança negativa $=0,63 \%$.

Tabela 7 - Valor da sensibilidade, especificidade, valor preditivo positivo e valor preditivo negativo, razão de verossimilhança positiva e razão de verossimilhança negativa da medida da relação cintura-quadril para diagnóstico de diabetes nos homens.

\begin{tabular}{l|l|c|c}
\hline & & \multicolumn{2}{c}{ Diabetes melito } \\
\hline & & Presente & Ausente \\
\hline \multirow{2}{*}{$\mathrm{RCQ}$} & Elevada & 1 & 9 \\
\cline { 2 - 4 } & Normal & 3 & 29 \\
\hline
\end{tabular}

Sensibilidade $=25 \%$; Especificidade $=76 \%$; Valor Preditivo positivo $=11,1 \%$; Valor preditivo negativo $=91 \%$; Razão de verossimilhança positiva $=1,0$; Razão de verossimilhança negativa $=0,99$.

As Tabelas 8 e 9 mostram os valores de sensibilidade, especificidade, valor preditivo positivo e valor preditivo negativo da medida da cintura e da relação cintura-quadril para o diagnóstico de dislipidemia. Os valores obtidos para a medida da cintura são sempre superiores aos valores obtidos para a relação cintura-quadril na população masculina. A razão de verossimilhança positiva é o valor mais elevado entre todos os obtidos no estudo e a razão de verossimilhança negativa também é o valor mais elevado.

$\mathrm{Na}$ amostra estudada o desempenho da relação cintura-quadril como preditor do diagnóstico de diabetes e dislipidemia é muito maior na população feminina enquanto o desempenho da medida da 
cintura isolada na população masculina para diagnóstico de diabetes e dislipidemia é muito maior do que na feminina. Esse dado pode indicar uma diferenciação no uso da medida da cintura e da RCQ para o diagnóstico de diabetes e dislipidemia nas populações feminina e masculina.

Tabela 8 - Valor da sensibilidade, especificidade, valor preditivo positivo e valor preditivo negativo, razão de verossimilhança positiva e razão de verossimilhança negativa da medida da cintura para diagnóstico de dislipidemia nos homens.

\begin{tabular}{l|c|c|c}
\hline & & \multicolumn{2}{|c}{$\begin{array}{c}\text { Dislipidemia } \\
\text { (Razão CT/HDL) }\end{array}$} \\
\hline & & Aumentada (>5) & Normal (<5) \\
\hline $\begin{array}{l}\text { Medida da } \\
\text { cintura (cm) }\end{array}$ & Elevada & 6 & 4 \\
\cline { 2 - 4 } & Normal & 4 & 28 \\
\hline
\end{tabular}

Sensibilidade $=60 \%$; Especificidade $=88 \%$; Valor Preditivo positivo $=60 \%$; Valor preditivo negativo $=88 \%$; Razão de verossimilhança positiva $=5$; Razão de verossimilhança negativa $=0.5$

Tabela 9 - Valor da sensibilidade, especificidade, valor preditivo positivo e valor preditivo negativo, razão de verossimilhança positiva e razão de verossimilhança negativa da medida da relação cintura-quadril para diagnóstico de dislipidemia nos homens.

\begin{tabular}{l|c|c|c}
\hline & & \multicolumn{2}{|c}{$\begin{array}{c}\text { Dislipidemia } \\
\text { (Razão CT/HDL) }\end{array}$} \\
\hline \multirow{2}{*}{ RCQ } & & Aumentada (>5) & Normal (<5) \\
\cline { 2 - 4 } & Elevada & 4 & 6 \\
\cline { 2 - 4 } & Normal & 6 & 26 \\
\hline
\end{tabular}

Sensibilidade $=40 \%$; Especificidade $=81 \%$; Valor Preditivo positivo $=40 \%$; Valor preditivo negativo $=81 \%$; Razão de verossimilhança positiva $=2,1$; Razão de verossimilhança negativa $=0,74$

\section{DISCUSSÃO}

Os resultados deste estudos mostram uma diferença no desempenho da medida da cintura e da relação cintura-quadril para o diagnóstico de diabetes melito e dislipidemia em relação aos sexos. $\mathrm{Na}$ amostra estudada, medida da cintura é um melhor preditor de diabetes e dislipidemia no sexo feminino enquanto a relação cintura-quadril é um melhor preditor de diabetes e dislipidemia no sexo masculino.

Embora o IMC seja a medida mais utilizada para avaliação da obesidade geral, na avaliação da obesidade localizada a relação cintura-quadril mostrou-se um índice mais preciso mostrando uma correlação positiva com o futuro aparecimento de doenças cardiovasculares, diabetes melito e seus fatores de risco $0^{5,6}$. Entretanto devido a dificuldade na padronização da medida do quadril surgiu a idéia de se utilizar somente a medida da cintura como um modo de se avaliar a obesidade localizada ${ }^{7}$. Estudos recentes analisaram o desempenho da medida da cintura e da relação cintura-quadril, mostrando que essas duas medidas podem ter um comportamento diferenciado em relação ao sexo e ao que exatamente está sendo avaliado por cada uma dessas medidas ${ }^{9,10,13}$.

Estudo tranversal realizado na Turquia mostrou uma relação entre idade, IMC e triglicérides como fator de risco independente da relação cintura-quadril em ambos os sexos, e a pressão diastólica somente no sexo feminino. Em relação a medida cintura, idade, IMC e pressão diastólica mostraram-se associados de forma independente para ambos os sexos, enquanto os níveis de colesterol se associaram somente ao sexo masculino, e os níveis de triglicérides, e da pressão sistólica somente nas mulheres. Houve uma correlação parcial entre na análise univariada entre a pressão arterial, os níveis de lipídeos e a medida da cintura e a relação cinturaquadril em ambos sexos, embora de melnor significância. As correlações foram sempre mais fortes para o sexo masculino em relação ao feminino e principalmente em relação à medida da cintura.

Molarius et al. ${ }^{9}$ estudaram 32000 homens e mulheres entre 25-64 anos que participaram do estudo MONICA, mostrando valores médios para a medida da cintura para homens e mulheres, respectivamente de $94-105 \mathrm{~cm}$ e $97-108 \mathrm{~cm}$. Para a mmedida da relação cintura-quadril, os valores médios foram respectivamente para homens e mulheres de 0,87 0,99 e $0,76-0,84$. Juntos, altura, IMC faixa etária e população estudada explicaram $80 \%$ da variabilidade na medida da cintura, sendo o IMC, o determinante principal. Valores semelhantes foram demonstrados em relação ã relação cintura-quadril mas a altura, IMC, faixa etária e população estudada contribuíram muito menos na determinação da variabilidade das medidas: $49 \%$ no sexo masculino e $30 \%$ no sexo feminino. $O$ estudo conclui que a medida da cintura $e$ a relação cintura-quadril refletem parâmetros diferentes: a medida da cintura refletiria muito mais 0 grau de sobrepeso de uma determinada população do que a $\mathrm{RCQ}^{9}$. Os mesmos autores, utilizando o mesmo banco de dados analisaram também o melhor ponto de corte para os valores da medida da cintura nas diferentes populações que compunham o estudo. Alterações nos pontos de corte para medida da cintura a diferentes níveis de IMC e RCQ, levam a modificações importantes da sensibilidade e especificidade da medida da cintura. Os autores concluem que os pontos de corte para a medida da 
cintura são população específicos e devem ser especificamente determinados.

O presente estudo mostrou um comportamento diferente da medida da cintura e da relação cintura quadril em homens e mulheres tanto como preditor do diabetes melito quanto da dislipidemia, representada por uma relação $\mathrm{CT} / \mathrm{HDL}$ elevada. $\mathrm{A}$ RCQ mostrou uma melhor sensibilidade, especifidade, VPP, VPN e razões de verossimilhança positiva e negativa em relação à medida da cintura no sexo feminino. O mesmoacontece em relação as mulheres para o diagnóstico de dislipidemia. Uma $R C Q$ tem um elevado valor preditivo negativo para diagnóstico tanto de diabetes quanto de dislipidemia. Em relação ao sexo masculino, ocorre o inverso. Tanto para o diagnóstico de diabetes quanto de dislipidemia, a medida da cintura apresenta um desempenho superior a da RCQ.

Possível explicação para esses achados seria que na mulher, devido ao predomínio da distribuição de gordura na região do quadril, é preferível que se utilize para o cálculo da obesidade abdominal, a RCQ, enquanto para o sexo masculino, com predomínio de obesidade abdominal, basta a utilização da medida da cintura.

Este estudo foi realizado em população que freqüentava o hospital, mas sem queixa específica de doença e que tinha procurado o serviço para consulta de promoção a saúde. O predomínio de mulheres traduz a maior freqüência do sexo feminino ao ambulatório. É um dos pucos estudos que discute o diferente comportamento da medida da cintura e da RCQ como preditores do diagnóstico de diabetes e dislipidemia em homens e mulheres.

Possíveis aplicações desse resultado consistem, em locais com poucos reecursos, como muitas regiões do país, na utilização da medida da cintura e da RCQ como triagem prévia para a seleção de pacientes que deverão se submeter a exames de glicemia e colesterol total e frações.

\section{CONCLUSÃO}

Na população estudada, a medida da cintura e a RCQ se comportam de maneira diferente no sexo masculino e feminino. A medida da cintura apresenta uma maior correlação com o aparecimento de diabetes e dislipidemia em homens, enquanto a RCQ apresenta um melhor desempenho em mulheres. Portanto, esses parâmetros devem ser utilizadas de forma diferenciada em homens e mulheres.

Calich, ALG Brunoni AR, Mansini R, Santo FRFE, Benseñor IM. Predictive value of the waist measurement and the waist-hip-ratio relationship on the diagnosis of diabetes mellitus and dyslipidemia. Rev Med (São Paulo) 2002 jan./ dez.;81(1/4):8-14.

ABSTRACT: Purpose: Obesity rates are increasing in Brazil. Waist measurement (WM) is a good measure to evaluate localized obesity and it is easier to perform when compared to wait-hip-ratio (WHR). However the effects of regional obesity were better described between WHR and cardiovascular risk factors. Aim: to test whether waist measurement alone is better than WHR to predict diabetes and high cholesterol. Methods: We analyzed positive and negative likelihood ratio (LR)of WM and WHR in a sample of 202 apparently healthy men and women aged 20-74 years as a predictive factor for diabetes and dislipidemia. Results: (1) For women, WHR was a better predictive factor for diabetes $\left(\mathrm{LHR}_{+}=2,5 \mathrm{e} \mathrm{LHR-}=0,2\right)$ and dislipidemia $(\mathrm{LHR}+=2,5, \mathrm{LHR}-=0,2)$ diagnosis than WM (diabetes, LHR $+=1,9 \mathrm{e}$ LHR- $=0,4$; dislipidemia, LHR+ = 1,6 e LHR- =0,6); (2) For men, WM was a better predictive factor for diabetes $(\mathrm{LHR}+=2,4 \mathrm{e} \mathrm{LHR-} \mathrm{=0,6)}$ and dislipidemia $(\mathrm{LHR}+=5$ e LHR- = 0,5) than for WHR (diabetes, LHR+ = 1,0 e LHR- = 0,9; dislipidemia, $\mathrm{LHR}+=2,1$ e LHR- = 0,7 diagnosis. Conclusion: WM and WHR have different behaviors in men and women.

KEYWORDS: Diabetes mellitus/diagnosis; Hyperlipidemia/diagnosis; Predictive value; Body constitution; Obesity/ etiology.

\section{REFERÊNCIAS}

1. Caraway WT, Watts NB. Carbohydrates. In: Tietz NW, editor. Fundamentals of clinical chemistry. 3rd ed. Philadelphia: WB Saunders; 1987. p.422-47.

2. Isaacsohn J, Black D, Troendle A, Orloff D. The impact of the National Cholesterol Education Program Adult Treatment Panel III guidelines on drug development Am J Cardiol 2002;89(5A):45C-9C.
3. Kisselbach $\mathrm{AH}$, Vydelingum N, Murray R, et al. Relation of body-fat distribution to metabolic complication in obesity. J Clin Endocrinol Metabol 1982;4:254-60.

4. Kleinbaum DG, Kupper LL, Morgernstern $\mathrm{H}$. Epidemiologic research. Principles and quantitative methods. Belmont, CA: Lifetime Learning 
Publications; 1982.

5. Krotkiewski M, Björntorp P, Sjostrom L, Smith U. Impact of obesity on metabolism in men and women: importance of regional adipose tissue distribution. J Clin Invest 1983;72:1150-62.

6. Larson B, Svarsudd K, Welin L, Wilhelmsen L, Björtorp P, Tibbin G. Abdominal adipose tissue distribution, obesity and risk of cardiovascular disease and death: 13 year follow-up of participants in the study of men born 1913. Br Med J 1984;288:1401-4.

7. Lean MEJ, Hans TS, Morrison C. Waist circumference as a measure for indicating need for weight management. Br Med J 1995;311:158-61.

8. Lotufo PA. Non-communicable diseases in Brazil: mortality patterns, morbidity studies and risk factors. Arch Latinoam Nutr 1997;47(2 Suppl 1):25-9.

9. Molarius A, Seidell JC, Sans S, Tuomilehto J, Kuulasmaa $\mathrm{K}$ for the WHO MONICA Project. Waist and hip circunferences, and waist-hip-ratio in 19 populations of the WHO MONICA Project. Int J Obes 1999;23:116-25.

10. Molarius A, Seidell JC, Sans S, Tuomilehto J, Kuulasmaa K for the WHO MONICA Project. Varying sensitivity of waist action levels to identify subjects with overweight or obesity in 19 populations of the WHO MONICA Project. J Clin Epidemiol 1999;52(12):1213-23.

11. Monteiro CA, Conde WL. A tendência secular da obesidade segundo estratos sociais: Nordeste e Sudeste do Brasil, 1957-1989-1997. Arq Bras Endocrinol Metab 1999;43:186-93.

12. Moss DW, Henderson AR, Kachmar JF. Enzymes. In: Tietz NW, editor. Fundamentals of clinical chemistry. 3rd ed. Philadelphia: WB Saunders; 1987. p.346-421. 13. Onat A, Sansoy V, Uysal Ö. Waist circumference and waist-to-hip ratio in Turkish adults: interrelation with other risk factors and association with cardiovascular disease. Int J Cardiol 1998;70:43-50.

14. Pouliot MC, Despres JP, Lemieux S, Moorjani S, Bouchard C, Tremblay A, et al. Waist circumference and abdominal sagittal diameter: best simple anthropometric indexes of abdominal visceral adipose tissue accumulation and related cardiovascular risk in men and women. Am J Cardiol 1994;73:460-8.

15. Sacks DB, Bruns DE, Goldstein DE, Maclaren NK, McDonald JM, Parrott M. Guidelines and recommendations for laboratory analysis in the diagnosis and management of diabetes mellitus. Diabetes Care 2002;25(4):750-86.

16. Stein EA. Lipids, lipoproteins, and apolipoproteins. In: Tietz NW, editor. Fundamentals of clinical chemistry. 3rd ed. Philadelphia: WB Saunders; 1987. p.448-81.

17. Velásquez-Meléndez G, Martins IS, Cervato AM, Fornés NS, Marucci M de FN, Coelho LT. Relationship between stature, overweight and central obesity in the adult population in São Paulo, Brazil. Int J Obes 1999;23:639-44.

18. Weltman A, Katch V. Preferencial use of casing (girth) measures for estimating body mass volume and density. J Appl Physiol 1975;38:560-3.

19. WHO Expert Committee. Physical status: the use and interpretation of anthropometry. Geneva: World Health Organization;1985. [WHO Technical Report Series no 854]. 